\title{
The Mathematical Model for Streptococcus suis Infection in Pig-Human Population with Humidity Effect
}

\author{
Inthira Chaiya ${ }^{1}$, Kamonchat Trachoo ${ }^{1}$, Kamsing Nonlaopon ${ }^{2}$ and Din Prathumwan ${ }^{2, *}$ \\ ${ }^{1}$ Department of Mathematics, Faculty of Science, Mahasarakham University, Mahasarakham, 44150, Thailand \\ ${ }^{2}$ Department of Mathematics, Faculty of Science, Khon Kaen University, Khon Kaen, 40002, Thailand \\ *Corresponding Author: Din Prathumwan. Email: dinpr@kku.ac.th \\ Received: 17 July 2021; Accepted: 27 September 2021
}

\begin{abstract}
In this paper, we developed a mathematical model for Streptococcus suis, which is an epidemic by considering the moisture that affects the infection. The disease is caused by Streptococcus suis infection found in pigs which can be transmitted to humans. The patients of Streptococcus suis were generally found in adults males and the elderly who contacted pigs or who ate uncooked pork. In human cases, the infection can cause a severe illness and death. This disease has an impact to the financial losses in the swine industry. In the development of models for this disease, we have divided the population into 7 related groups which are susceptible pig compartment, infected pig compartment, quarantined pig compartment, recovered pig compartment, susceptible human compartment, infected human compartment, and recovered human compartment. After that, we use this model and a quarantine strategy to analyze the spread of the infection. In addition, the basic reproduction number $R_{0}$ is determined by using the next-generation matrix which can analyze the stability of the model. The numerical simulations of the proposed model are illustrated to confirm the results from theorems. The results showed that there is an effect from moisture to the disease transmission. When the moisture increases the disease infection also increases.
\end{abstract}

Keywords: Streptococcus suis; mathematical model; stability analysis; infectious disease; reproduction number

\section{Introduction}

Nowadays, one of the major problems in the world is zoonotic pathogen such as Rift Valley fever, SARS, pandemic influenza H1N1 2009, Yellow fever, Avian Influenza (H5N1) and (H7N9), West Nile virus [1], the Middle East respiratory syndrome coronavirus (MERSCoV) [2], and novel coronavirus (COVID-19) [3]. Streptococcus suis is an infectious agent which is widely found in pigs around the world. This pathogen is generally found in the upper respiratory tract, genital, and alimentary tracts of the pigs [4,5]. Streptococcus suis is a Gram-positive bacterium and a facultative anaerobic bacterium which is a primary agent of sepsis and meningitis in pigs $[6,7]$. In pig farms, the disease can transmit

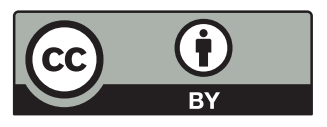

This work is licensed under a Creative Commons Attribution 4.0 International License, which permits unrestricted use, distribution, and reproduction in any medium, provided the original work is properly cited. 
from pig to pig rapidly. The reported rate of mortality in pigs from Streptococcus suis is about $20 \%$ [8]. It can classify at least 35 serotypes, for examples, serotypes 1 to 31, 33, and 1/2 [9,10]. Serotypes 1 to 9 and 14 are most commonly found in pigs around the world [11,12].

In 1968, the first human who was infected with Streptococcus suis was found in Denmark [4,5]. This situation confirms that Streptococcus suis can transmit from pig to human. Serotype 2 is reported that it is frequently found in human infections [13,14], but in some cases, the infections are caused by other serotypes. Kerdsin et al. reported that serotypes 2 and 14 are involved in human cases that found in Thailand $[15,16]$. Dutkiewicz et al. presented that disease found in human caused by $S$. suis serotypes 2, 4, 5, 14, 16, 21 and 24 [12]. S. suis serotype 9 which is the most common found in pigs in Europe was found the first case infection in human in May 2013 locating in northern of Thailand [17]. In addition, Hatrongiit et al. reported the first case of serotype 31 in human [18] in the central region of Thailand. The common symptoms of this disease in human are fever, headache, meningitis, septicemia, arthritis, pneumonia, and hearing loss [19].

To prevent the spread of the disease, the mathematical models become powerful tools for describing the dynamics of the disease. They can forecast the future behaviors of the disease with many assumptions. The solutions of the model can be simulated satisfying the given parameters from the hypothesis. Recently, many mathematical models have been used to describe the behavior of infectious disease [20-24]. Rahman et al. proposed the nonlinear SEITR fractional order model of tuberculosis disease by using Atangana-Baleanu derivative which consists of 5 population groups [25]. Arfan et al. [26] presented the fractional order model to predict the dynamic of tumor with drug intervention. The nonlinear fractional order mathematical model for predicting the dynamic of COVID-19 was created by Shah et al. [27]. Almuqrin et al. proposed the fractional model for forecasting the transmission of Ebola virus in bats population by using Atangana-Baleanu fractional derivative [28]. Srivastava et al. improved the mathematical model for studying the dynamic of diabetes patients [29]. Alzaid et al. [30] studied the dynamic of HIV-1 infection by using the mathematical model and showed that the mathematical model is efficient. The fractional order model of HIV with source term was proposed by Shah et al. to consider the behavior of CD4 + T-cells which depending on the concentration of the viral load [31]. The symmetry and asymmetry concepts can be linked to the epidemic model [32-34].

A number of research studied the disease from Streptococcus suis on epidemiology and medicine. However, a few pieces of the research proposed and studied the mathematical model for Streptococcus suis. Shen et al. [35] proposed the SIQRW model to explore the outbreaks of S. Suis. Giang et al. proposed the stochastic model and SEI model to predict the behavior of the disease and fitted the model parameters with collected data [9]. The proposed models consider the transmission on pig population only. However, to our knowledge, no research considering the disease transmission between pigs and humans for Streptococcus suis.

In this paper, we propose a mathematical model to describe the disease transmission of Streptococcus suis in humans and pigs. The novelty of this work is the improved mathematical model from one species to two species population. We classify the population of pigs and humans into 4 classes and 3 classes, respectively. This work contains the analysis of the model and numerical simulations to study the dynamic behavior of the disease. We also find the basic reproduction numbers to explain the rapid transmission and study the stability of the solution of the model.

This paper is constructed as follows; In Section 2, we present the mathematical model which expresses the Streptococcus suis transmission. The analysis of the proposed model is shown in Section 3 which includes a basic reproduction number, equilibrium points, and their stability. The numerical simulations are given in Section 4 and follow by a conclusion in Section 5. 


\section{Model Formulation}

In this section, we describe the model formulation of Streptococcus suis disease transmission in humans and pigs. Based on the classical epidemiology model, we propose a new generalized model of Streptococcus suis infection which is SIQR-SIR model. There are some facts about the environmental factors such as temperature and relative humidity [36-38]. So, we consider the effect of the moisture in the air for disease transmission in the pig farm.

The investigated population is divided into two subpopulations. These are pig population and human population. Then, both subpopulations of pigs and human are separated into four classes and three classes, respectively. These are a pig susceptible class $\left(S_{p}\right)$, pig infectious class $\left(I_{p}\right)$, pig isolated class $\left(Q_{p}\right)$, pig recovery class $\left(R_{p}\right)$, human susceptible class $\left(S_{h}\right)$, human infectious class $\left(I_{h}\right)$, and human recovery class $\left(R_{p}\right)$. We assume that the total population at time $t$ is $N(t)$. We have that

$S_{p}(t)+I_{p}(t)+Q_{p}(t)+R_{p}(t)=N_{p}(t)$,

$S_{h}(t)+I_{h}(t)+R_{h}(t)=N_{h}(t)$,

and

$S_{p}(t)+I_{p}(t)+Q_{p}(t)+R_{p}(t)+S_{h}(t)+I_{h}(t)+R_{h}(t)=N_{p}(t)+N_{h}(t)=N(t)$,

where $N_{p}(t)$ is the total population of pigs, and $N_{h}(t)$ is the total population of human at time $t$.

We can write the dynamic equations for $N_{p}(t)$ and $N_{h}(t)$ as the following.

$\frac{d N_{p}}{d t}=\frac{d S_{p}}{d t}+\frac{d I_{p}}{d t}+\frac{d Q_{p}}{d t}+\frac{d R_{p}}{d t}$

$\frac{d N_{h}}{d t}=\frac{d S_{h}}{d t}+\frac{d I_{h}}{d t}+\frac{d R_{h}}{d t}$.

The new infection in pigs can be described . where $\beta_{1}$ is the transmission coefficient per unit of time per pig in the susceptible class contact with infectious class [39]. The new infection in human can be described by $\beta_{2} S_{h} I_{p}+\beta_{3} S_{h} I_{h}$ where $\beta_{2}$ is the transmission coefficient per unit of time per person in the susceptible class in contact with pig infectious class, $\beta_{3}$ is the transmission coefficient per unit of time per person in the susceptible class contact with the infectious class. In our model, we investigate the relative humidity for considering the infection of this disease in pigs. Then, the new infection in pigs can be expressed by $\beta_{1} M S_{p} I_{p}$ instead of $\beta_{1} S_{p} I_{p}$, where $M$ is the moisture in the air (relative humidity). In addition, we assume that the disease cannot be transmitted from human to pigs. Throughout this paper, we assume that all parameters are positive constants.

The model of disease transmission by Streptococcus suis can be represented by the system of differential equations as follows:

$$
\begin{aligned}
& \frac{d S_{p}}{d t}=N_{1}-b S_{p}-\beta_{1} M S_{p} I_{p}, \\
& \frac{d I_{p}}{d t}=\beta_{1} M S_{p} I_{p}-a I_{p}-b I_{p}-\delta I_{p}, \\
& \frac{d Q_{p}}{d t}=\delta I_{p}-a Q_{p}-b Q_{p}-\epsilon Q_{p},
\end{aligned}
$$




$$
\begin{aligned}
& \frac{d R_{p}}{d t}=\epsilon Q_{p}-b R_{p}, \\
& \frac{d S_{h}}{d t}=N_{2}-\beta_{2} S_{h} I_{p}-\beta_{3} S_{h} I_{h}-\mu S_{h}, \\
& \frac{d I_{h}}{d t}=\beta_{2} S_{h} I_{p}+\beta_{3} S_{h} I_{h}-\alpha I_{h}-\gamma I_{h}-\mu I_{h}, \\
& \frac{d R_{h}}{d t}=\gamma I_{h}-\mu R_{h},
\end{aligned}
$$

where $b$ is the pig removal rate, $a$ is the pig death rate induced by the disease, $\delta$ is the rate from infectious class to isolated class in pigs, $e$ is the transition rate from isolated class to recovery class, $\mu$ is the human natural death rate, $\alpha$ is the death rate induced by disease, and $\gamma$ is the transition rate from infectious class to recovery class. The model form is valid only if $a \leq b$ and $\alpha \leq \mu$.

Eq. (1a) expresses the rate of change of the population of susceptible pig. The number of susceptible pigs increases only via birth $N_{1}$ described in the first term on the right-hand side. On the other hand, the population of them decreases due to the removal rate of pig $(b)$, transmission of Streptococcus suis from infected pig to susceptible pig $\left(\beta_{1}\right)$ and moisture effect $(M)$.

Eq. (1b) expresses the rate of change of the population of the infected pig. On the right-hand side, the first term represents the number of infected pigs increases due to susceptible pig becoming infected pig with the rate $\beta_{1} M$ followed by the negative effects of disease death rate $(a)$, removal rate $(b)$, and isolated rate $(\delta)$.

Eq. (1c) expresses the rate of change of the population of the isolated pig. On the right-hand side, the first term represents the increase in the population of isolated pig due to the isolation of infected pig with the rate $\delta$ followed by the negative effects of disease death rate $(a)$, removal rate $(b)$, and recovered rate $(\epsilon)$.

Eq. (1d) expresses the rate of change of the population of the recovered pig. On the right-hand side, the first term represents the increase in the population of the recovered pig due to treatment with the rate $\epsilon$, and the last term represents the removal rate $(b)$ of pigs.

Eq. (1e) expresses the rate of change of the population of susceptible human. The number of susceptible pigs increases only via birth $N_{2}$ described in the first term on the right-hand side. On the other hand, the population of them decreases due to the transmission of Streptococcus suis from infected pig to susceptible human $\left(\beta_{2}\right)$ and infected human to susceptible human $\left(\beta_{3}\right)$. The last term represents the natural death rate $(\mu)$ of human.

Eq. (1f) expresses the rate of change of the population of the infected human. On the righthand side, the first term and second term represent the number of the infected human increases due to susceptible human becoming infected human by contact with the infected pigs $\left(\beta_{2}\right)$ and the infected human $\left(\beta_{3}\right)$, respectively. The remaining terms are the negative effects of disease death rate $(\alpha)$, recovered rate $(\gamma)$, and natural death rate $(\mu)$.

Eq. (1g) expresses the rate of change of the population of recovered human. On the right-hand side, the first term represents the increase in the population of recovered human due to treatment with the rate $(\gamma)$, and the last term represents the natural death rate $(\mu)$ of human 
Let $S_{p}(0)=S_{p 0}, I_{p}(0)=I_{p 0}, \quad Q_{p}(0)=Q_{p 0}, R_{p}(0)=R_{p 0}, S_{h}(0)=S_{h 0}, I_{h}(0)=I_{h 0}, R_{h}(0)=R_{h 0}$. A flowchart of the SIQR-SIR model of pigs and human which describe by the system (1) is shown in Fig 1.

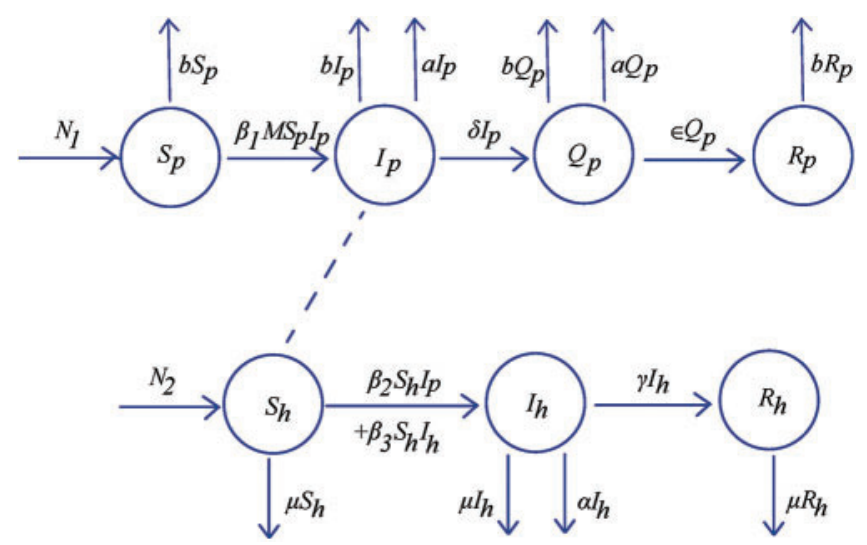

Figure 1: Flowchart of SIQR-SIR (Susceptible-Infectious-Isolation-Recovery-Susceptible-InfectiousRecovery) model for human and pigs

The values of population classes $S_{p}(t), I_{p}(t), Q_{p}(t), R_{p}(t), S_{h}(t), I_{h}(t), R_{h}(t)$ at time $t$ is nonnegative numbers.

Let

$N(t)=S_{p}(t)+I_{p}(t)+Q_{p}(t)+R_{p}(t)+S_{h}(t)+I_{h}(t)+R_{h}(t)$,

and $c=\min \{b, \mu\}$. Then we obtain

$$
\begin{aligned}
\frac{d N}{d t} & =\frac{d S_{p}}{d t}+\frac{d I_{p}}{d t}+\frac{d Q_{p}}{d t}+\frac{d R_{p}}{d t}+\frac{d S_{h}}{d t}+\frac{d I_{h}}{d t}+\frac{d R_{h}}{d t} \\
& =N_{1}+N_{2}-a\left(I_{p}+Q_{p}\right)-b\left(S_{p}+I_{p}+Q_{p}+R_{p}\right)-\alpha I_{h}-\mu\left(S_{h}+I_{h}+R_{h}\right) \\
& \leq N_{1}+N_{2}-c N
\end{aligned}
$$

and

$N(t) \leq \frac{N_{1}+N_{2}}{c}+\left(N(0)-\frac{N_{1}+N_{2}}{c}\right) e^{-c t}$.

Therefore, the positive invariant of the system (1) is

$\Omega=\left\{\left(S_{p}, I_{p}, Q_{p}, R_{p}, S_{h}, I_{h}, R_{h}\right) \in R_{+}^{7}: N \leq \frac{N_{1}+N_{2}}{c}\right\}$. 


\section{Model Analysis}

\subsection{Equilibria}

The equilibria are obtained by setting all equations of the system (1) to be zero.

$N_{1}-b S_{p}-\beta_{1} M S_{p} I_{p}=0$

$\beta_{1} M S_{p} I_{p}-a I_{p}-b I_{p}-\delta I_{p}=0$

$\delta I_{p}-a Q_{p}-b Q_{p}-\epsilon Q_{p}=0$,

$\epsilon Q_{p}-b R_{p}=0$

$N_{2}-\beta_{2} S_{h} I_{p}-\beta_{3} S_{h} I_{h}-\mu S_{h}=0$

$\beta_{2} S_{h} I_{p}+\beta_{3} S_{h} I_{h}-\alpha I_{h}-\gamma I_{h}-\mu I_{h}=0$,

$\gamma I_{h}-\mu R_{h}=0$.

By solving the system (4), We get three equilibrium points:

i) Disease-free equilibrium

$$
E_{1}=\left(\frac{N_{1}}{b}, 0,0,0, \frac{N_{2}}{\mu}, 0,0\right) .
$$

ii) Pig disease free equilibrium

$$
E_{2}=\left(\frac{N_{1}}{b}, 0,0,0, \frac{A}{\beta_{3}}, \frac{\beta_{3} N_{2}-\mu A}{\beta_{3} A}, \frac{\gamma}{\mu} \frac{\beta_{3} N_{2}-\mu A}{\beta_{3} A}\right),
$$

where $A \equiv \alpha+\gamma+\mu$.

Note that, $E_{2}$ exists if $\beta_{3} N_{2}-\mu A>0$.

iii) The endemic equilibrium

$$
E^{*}=\left(S_{p}^{*}, I_{p}^{*}, Q_{p}^{*}, R_{p}^{*}, S_{h}^{*}, I_{h}^{*}, R_{h}^{*}\right)
$$

where

$S_{p}^{*}=\frac{B}{\beta_{1} M}$,

$I_{p}^{*}=\frac{\beta_{1} M N_{1}-b B}{\beta_{1} B M}$,

$Q_{p}^{*}=\frac{\delta I_{p}^{*}}{(a+b+\epsilon)}$, 
$R_{p}^{*}=\frac{\epsilon \delta I_{p}^{*}}{b(a+b+\epsilon)}$,

$I_{h}^{*}=\frac{1}{2 \beta_{3} A}\left(X+\sqrt{X^{2}+4 A \beta_{2} \beta_{3} N_{2} I_{p}^{*}}\right)$,

$S_{h}^{*}=\frac{N_{2}}{\beta_{2} I_{p}^{*}+\beta_{3} I_{h}^{*}+\mu}$,

$R_{h}^{*}=\frac{\gamma I_{h}^{*}}{\mu}$

with $X \equiv \beta_{3} N_{2}-A\left(\beta_{2} I_{p}^{*}+\mu\right)$ and $B \equiv a+b+\delta$.

Note that, $E^{*}$ exists if $\beta_{1} M N_{1}-b B>0$.

\subsection{Basic Reproduction Number}

The basic reproduction number is the expected number of secondary cases produced by a single infection in a completely susceptible population. To compute the basic reproduction number and to study the local stability of the equilibrium, we use the next generation matrix method [40,41]. We define $\mathbf{x}^{\prime}=f(\mathbf{x})-v(\mathbf{x})$, where $\mathbf{x}=\left[I_{p}, Q_{p}, I_{h}\right]^{T}$ where $f(\mathbf{x})$ is the matrix of new infection terms, and $v(\mathbf{x})$ is the matrix of transfer terms into compartment and out of compartment as:

$f=\left[\begin{array}{c}\beta_{1} M S_{p} I_{p} \\ 0 \\ \beta_{2} S_{h} I_{p}+\beta_{3} S_{h} I_{h}\end{array}\right], v=\left[\begin{array}{c}a I_{p}+b I_{p}+\delta I_{p} \\ \delta I_{p}+b Q_{p}+\epsilon Q_{p} \\ \alpha I_{h}+\gamma I_{h}+\mu I_{h}\end{array}\right]$.

The corresponding Jacobian matrices are

$F=\left[\begin{array}{ccc}\beta_{1} M S_{p} & 0 & 0 \\ 0 & 0 & 0 \\ \beta_{2} S_{h} & 0 & \beta_{3} S_{h}\end{array}\right], V=\left[\begin{array}{ccc}B & 0 & 0 \\ -\delta & a+b+\epsilon & 0 \\ 0 & 0 & A\end{array}\right]$

Then, we obtain

$$
\begin{aligned}
F V^{-1}= & {\left.\left[\begin{array}{ccc}
\frac{\beta_{1} M S_{p}}{B} & 0 & 0 \\
0 & 0 & 0 \\
\frac{\beta_{2} S_{h}}{B} & 0 & \frac{\beta_{3} S_{h}}{A}
\end{array}\right]\right|_{E_{1}} } \\
& =\left[\begin{array}{ccc}
\frac{\beta_{1} M N_{1}}{b B} & 0 & 0 \\
0 & 0 & 0 \\
\frac{\beta_{2} N_{2}}{\mu B} & 0 & \frac{\beta_{3} N_{2}}{\mu A}
\end{array}\right]
\end{aligned}
$$

We obtain three eigenvalues of $F V^{-1}$ as:

$\lambda_{1}=0, \lambda_{2}=\frac{\beta_{3} N_{2}}{\mu A}, \lambda_{3}=\frac{\beta_{1} M N_{1}}{b B}$.

Hence, the spectral radii are $R_{01}=\frac{\beta_{3} N_{2}}{\mu A}$, and $R_{02}=\frac{\beta_{1} M N_{1}}{b B}$. 


\subsection{The Stability of Disease-Free Equilibrium $\left(E_{1}\right)$}

Theorem 1. The disease-free equilibrium point $\left(E_{1}\right)$ is locally asymptotically stable if $R_{01}<1$ and $R_{02}<1$.

Proof. The model system (1) has the Jacobian matrix at the point $E_{1}$ as:

$J\left(E_{1}\right)=\left[\begin{array}{lllllll}-b & -\frac{\beta_{1} M N_{1}}{b^{b}} & 0 & 0 & 0 & 0 & 0 \\ 0 & \frac{\beta_{1} M N_{1}}{b}-B & 0 & 0 & 0 & 0 & 0 \\ 0 & \delta & -(a+b+\epsilon) & 0 & 0 & 0 & 0 \\ 0 & 0 & \epsilon & -b & 0 & 0 & 0 \\ 0 & -\frac{\beta_{2} N_{2}}{\mu} & 0 & 0 & -\mu & -\frac{\beta_{3} N_{2}}{\mu} & 0 \\ 0 & \frac{\beta_{2} N_{2}}{\mu} & 0 & 0 & 0 & \frac{\beta_{3} N_{2}}{\mu}-A & 0 \\ 0 & 0 & 0 & 0 & 0 & \gamma & -\mu\end{array}\right]$

The corresponding eigenvalues of $J\left(E_{1}\right)$ are

$\lambda_{1}=\lambda_{2}=-b, \lambda_{3}=\lambda_{4}=-\mu, \lambda_{5}=-(a+b+\epsilon)$,

$\lambda_{6}=\frac{\beta_{1} M N_{1}}{b B}-B=B\left(R_{02}-1\right)$,

$\lambda_{7}=\frac{\beta_{3} N_{2}}{\mu}-A=A\left(R_{01}-1\right)$.

Note that $\lambda_{6}$ is negative if $R_{02}<1$ and $\lambda_{7}$ is negative if $R_{01}<1$.

Therefore, the disease-free equilibrium $\left(E_{1}\right)$ of the model system (1) is locally asymptotically stable due to all negative real part eigenvalues if $R_{01}<1$ and $R_{02}<1$.

\subsection{The Stability of Pig Disease Free Equilibrium $\left(E_{2}\right)$}

Theorem 2. The pig disease free equilibrium $\left(E_{2}\right)$ is locally asymptotically stable if $R_{01}>1$ and $R_{02}<1$.

Proof. The pig disease-free equilibrium

$E_{2}=\left(\frac{N_{1}}{b}, 0,0,0, \frac{A}{\beta_{3}}, \frac{\beta_{3} N_{2}-\mu A}{\beta_{3} A}, \frac{\gamma}{\mu} \frac{\beta_{3} N_{2}-\mu A}{\beta_{3} A}\right)$

exists and is positive if $R_{01}>1$.

The model system (1) has the Jacobian matrix at the point $E_{2}$ as:

$$
J\left(E_{2}\right)=\left[\begin{array}{lllllll}
-b & -\frac{\beta_{1} M N_{1}}{b^{b}} & 0 & 0 & 0 & 0 & 0 \\
0 & \frac{\beta_{1} M N_{1}}{b}-B & 0 & 0 & 0 & 0 & 0 \\
0 & \delta & -(a+b+\epsilon) & 0 & 0 & 0 & 0 \\
0 & 0 & \epsilon & -b & 0 & 0 & 0 \\
0 & -\frac{\beta_{2} N_{2}}{\mu R_{01}} & 0 & 0 & -\mu R_{01} & -A & 0 \\
0 & \frac{\beta_{2} N_{2}}{\mu R_{01}} & 0 & 0 & \mu\left(R_{01}-1\right) & 0 & 0 \\
0 & 0 & 0 & 0 & 0 & \gamma & -\mu
\end{array}\right]
$$


CMC, 2022, vol.71, no.2

The corresponding eigenvalues of $J\left(E_{2}\right)$ are

$\lambda_{1}=\lambda_{2}=-b, \lambda_{3}=-\mu, \lambda_{4}=-(a+b+\epsilon)$,

$\lambda_{5}=\frac{\beta_{1} M N_{1}}{b}-B=B\left(R_{02}-1\right)$,

$\lambda_{6}=\frac{-\mu R_{01}+\sqrt{\mu^{2} R_{01}^{2}-4 \mu A\left(R_{01}-1\right)}}{2}$,

$\lambda_{7}=\frac{-\mu R_{01}-\sqrt{\mu^{2} R_{01}^{2}-4 \mu A\left(R_{01}-1\right)}}{2}$.

Note that $\lambda_{5}$ is negative if $R_{02}<1$ while $\lambda_{6}$ and $\lambda_{7}$ have negative real part if $R_{01}>1$.

Therefore, the pig disease free equilibrium $\left(E_{2}\right)$ of the system $(1)$ is locally asymptotically stable if $R_{01}>1$ and $R_{02}<1$.

\subsection{The Stability of Endemic Equilibrium $\left(E^{*}\right)$}

Theorem 3. The endemic equilibrium $\left(E^{*}\right)$ is locally asymptotically stable if $R_{01}<1$ and $R_{02}>1$.

Proof. The endemic equilibrium

$E^{*}=\left(S_{p}^{*}, I_{p}^{*}, Q_{p}^{*}, R_{p}^{*}, S_{h}^{*}, I_{h}^{*}, R_{h}^{*}\right)$,

where

$S_{p}^{*}=\frac{N_{1}}{b R_{02}}$

$I_{p}^{*}=\frac{b\left(R_{02}-1\right)}{\beta_{1} M}$

$Q_{p}^{*}=\frac{\delta I_{p}^{*}}{(a+b+\epsilon)}$

$R_{p}^{*}=\frac{\epsilon \delta I_{p}^{*}}{b(a+b+\epsilon)}$

$I_{h}^{*}=\frac{1}{2 \beta_{3} A}\left(X+\sqrt{X^{2}+4 A \beta_{2} \beta_{3} N_{2} I_{p}^{*}}\right)$

$S_{h}^{*}=\frac{N_{2}}{\beta_{2} I_{p}^{*}+\beta_{3} I_{h}^{*}+\mu}$

$R_{h}^{*}=\frac{\gamma I_{h}^{*}}{\mu}$

exists and is positive if $R_{02}>1$. 
The model system (1) has the Jacobian matrix at the point $E^{*}$ as:

$$
J\left(E_{2}\right)=\left[\begin{array}{lllllll}
-b-\Psi_{1} & -\Psi_{2} & 0 & 0 & 0 & 0 & 0 \\
\Psi_{1} & \Psi_{2}-B & 0 & 0 & 0 & 0 & 0 \\
0 & \delta & -(a+b+\epsilon) & 0 & 0 & 0 & 0 \\
0 & 0 & \epsilon & -b & 0 & 0 & 0 \\
0 & -\beta_{2} S_{h}^{*} & 0 & 0 & -\Psi_{3}-\mu & -\beta_{3} S_{h}^{*} & 0 \\
0 & \beta_{2} S_{h}^{*} & 0 & 0 & \Psi_{3} & \beta_{3} S_{h}^{*}-A & 0 \\
0 & 0 & 0 & 0 & 0 & \gamma & -\mu
\end{array}\right]
$$

where

$\Psi_{1}=\frac{\beta_{1} M N_{1}\left(R_{02}-1\right)}{B R_{02}}, \Psi_{2}=\frac{M \beta_{1} N_{1}}{b R_{02}}, \Psi_{3}=\frac{-\beta_{2} N_{1}\left(R_{02}-1\right)}{B R_{02}}+\beta_{3} I_{h}^{*}$.

The corresponding eigenvalues of $J\left(E^{*}\right)$ are

$\lambda_{1}=-b, \lambda_{2}=-\mu, \lambda_{3}=-(a+b+\epsilon)$,

$\lambda_{4}=\frac{-\left[\beta_{1} M\left(I_{p}^{*}-S_{p}^{*}\right)+B+b\right]+Y_{1}}{2}$,

$\lambda_{5}=\frac{-\left[\beta_{1} M\left(I_{p}^{*}-S_{p}^{*}\right)+B+b\right]-Y_{1}}{2}$,

$\lambda_{6}=\frac{-\left[\beta_{2} I_{p}^{*}+\beta_{3}\left(I_{h}^{*}-S_{h}^{*}\right)+A+\mu\right]+Y_{2}}{2}$,

$\lambda_{7}=\frac{-\left[\beta_{2} I_{p}^{*}+\beta_{3}\left(I_{h}^{*}-S_{h}^{*}\right)+A+\mu\right]-Y_{2}}{2}$,

where

$Y_{1}=\sqrt{\left[\beta_{1} M\left(I_{p}^{*}-S_{p}^{*}\right)+B+b\right]^{2}-4\left[\beta_{1} M\left(B I_{p}^{*}-b S_{p}^{*}\right)+B b\right]}$,

and

$Y_{2}=\sqrt{\left[\beta_{2} I_{p}^{*}+\beta_{3}\left(I_{h}^{*}-S_{h}^{*}\right)+A+\mu\right]^{2}-4\left[\beta_{2} A I_{p}^{*}+\beta_{3} A I_{h}^{*}+\mu\left(A-\beta_{3} S_{h}^{*}\right)\right]}$.

Next, we consider

$$
\begin{aligned}
\beta_{1} M\left(I_{p}^{*}-S_{p}^{*}\right)+B+b & =\beta_{1} M\left(\frac{b\left(R_{02}-1\right)}{\beta_{1} M}-\frac{N_{1}}{b R_{02}}\right)+B+b \\
& =b\left(R_{02}-1\right)-\frac{\beta_{1} M N_{1}}{b R_{02}}+B+b
\end{aligned}
$$


CMC, 2022, vol.71, no.2

$$
\begin{aligned}
& =b R_{02} \\
& >0,
\end{aligned}
$$

and

$$
\begin{aligned}
\beta_{1} M\left(B I_{p}^{*}-b S_{p}^{*}\right)+B b & =\beta_{1} M\left(\frac{B b\left(R_{02}-1\right)}{\beta_{1} M}-\frac{b N_{1}}{b R_{02}}\right)+B b \\
& =b B\left(R_{02}-1\right)-\frac{b \beta_{1} M N_{1}}{b R_{02}}+B b \\
& =B b\left(R_{02}-1\right) .
\end{aligned}
$$

We obtain that $\lambda_{4}$ and $\lambda_{5}$ has negative real part if $R_{02}>1$.

Then, let us consider

$$
\begin{aligned}
& \beta_{2} I_{p}^{*}+\beta_{3}\left(I_{h}^{*}-S_{h}^{*}\right)+A+\mu \\
= & \beta_{2} I_{p}^{*}+\beta_{3}\left(I_{h}^{*}-\frac{N_{2}}{\beta_{2} I_{p}^{*}+\beta_{3} I_{h}^{*}+\mu}\right)+A+\mu \\
= & \frac{\beta_{3}^{2} I_{h}^{* 2}+\left[2 \beta_{2} \beta_{3} I_{p}^{*}+2 \mu \beta_{3}+\beta_{3} A\right] I_{h}^{*}+\beta_{2}^{2} I_{p}^{* 2}+(A+2 \mu) \beta_{2} I_{p}^{*}+\mu(A+\mu)-\beta_{3} N_{2}}{\beta_{2} I_{p}^{*}+\beta_{3} I_{h}^{*}+\mu} \\
= & \frac{\left[\beta_{3}^{2}+\beta_{3} A\right] I_{h}^{* 2}+\left[2 \beta_{2} \beta_{3} I_{p}^{*}+\beta_{3}\left(2 \mu+A-N_{2}\right)+A\left(\beta_{2} I_{p}^{*}+\mu\right)\right] I_{h}^{*}}{\beta_{2} I_{p}^{*}+\beta_{3} I_{h}^{*}+\mu} \\
& +\frac{\beta_{2} I_{p}^{*}\left(\beta_{2} I_{p}^{*}+A+2 \mu-N_{2}\right)+\mu(A+\mu)-\beta_{3} N_{2}}{\beta_{2} I_{p}^{*}+\beta_{3} I_{h}^{*}+\mu} .
\end{aligned}
$$

Since $I_{h}^{*}=\frac{1}{2 \beta_{3} A}\left(X+\sqrt{X^{2}+4 A \beta_{2} \beta_{3} N_{2} I_{p}^{*}}\right)$, it follows that $\beta_{3} A I_{h}^{* 2}-\left[\beta_{3} N_{2}-A\left(\beta_{2} I_{p}^{*}+\mu\right)\right] I_{h}^{*}-\beta_{2} N_{2} I_{p}^{*}=0$.

Then, we get

$$
\begin{aligned}
\beta_{2} I_{p}^{*} & +\beta_{3}\left(I_{h}^{*}-S_{h}^{*}\right)+A+\mu \\
& =\frac{\beta_{3}^{2} I_{h}^{* 2}+\left[2 \beta_{2} \beta_{3} I_{p}^{*}+\beta_{3}(2 \mu+A)\right] I_{h}^{*}+\beta_{2} I_{p}^{*}\left(\beta_{2} I_{p}^{*}+A+2 \mu\right)+\mu^{2}+\mu A\left(1-R_{01}\right)}{\beta_{2} I_{p}^{*}+\beta_{3} I_{h}^{*}+\mu} .
\end{aligned}
$$

If $R_{01}<1, I_{p}^{*}$ and $I_{h}^{*}$ are positive i.e., $R_{02}>1$, then we obtain $\beta_{2} I_{p}^{*}+\beta_{3}\left(I_{h}^{*}-S_{h}^{*}\right)+A+\mu>0$. 
Thus, the eigenvalues $\lambda_{5}$ and $\lambda_{6}$ have a negative real part if $R_{01}<1$ and $R_{02}>1$.

Therefore, the endemic equilibrium $\left(E^{*}\right)$ of the model system (1) is locally asymptotically stable if $R_{01}<1$ and $R_{02}>1$.

We can draw the bifurcation diagram to describe the basic reproduction numbers and stability as shown in Fig. 2.

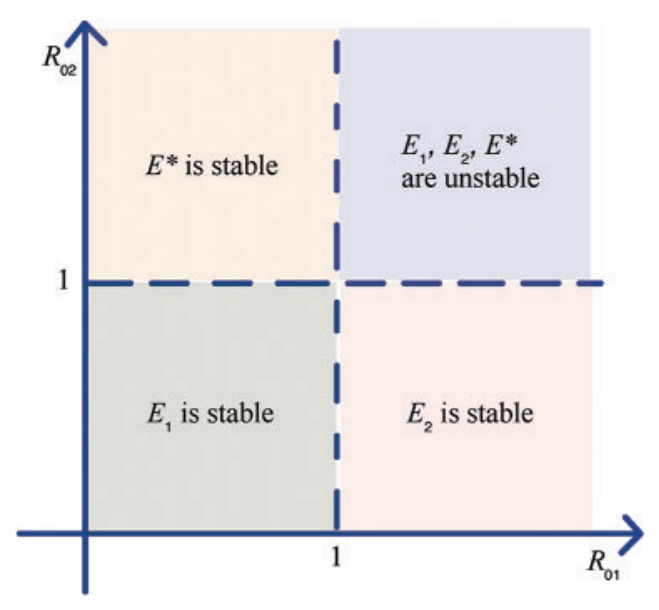

Figure 2: The bifurcation region

\section{Numerical Examples and Discussion} values:

The numerical results of the system (1) are computed by using MATLAB with the given initial $S_{p}(0)=4057, I_{p}(0)=1000, Q_{p}(0)=0, R_{p}(0)=0, S_{h}(0)=50000, I_{h}(0)=1000, R_{h}(0)=0$.

The numerical results of the system (1) with the parameter values as shown in Tab. 1. Note that the parameters and initial values are obtained from data in [35] and [42].

Table 1: Parameter values of the system (1)

\begin{tabular}{lll}
\hline Parameter & Value & Source \\
\hline$N_{1}$ & 3275 & Taken from [35] \\
$b$ & 0.75 & Taken from [35] \\
$\beta_{1}$ & 0.000365 & Taken from [35] \\
$N_{2}$ & 500 & Calculated \\
$\beta_{3}$ & 0.00465 & Assumed \\
\hline
\end{tabular}

The solution trajectories tend to the disease-free equilibrium $\left(E_{1}\right)$ which satisfy Theorem 1 with the remaining parameter values $\mu=0.9, \alpha=0.9, \gamma=0.9, M=0.9, a=0.9, \beta_{2}=0.1$, and $\delta=0.9$ as 
shown in Fig. 3. The calculated reproduction numbers of this case are $R_{01}=0.9568<1$ and $R_{02}=$ $0.5625<1$.

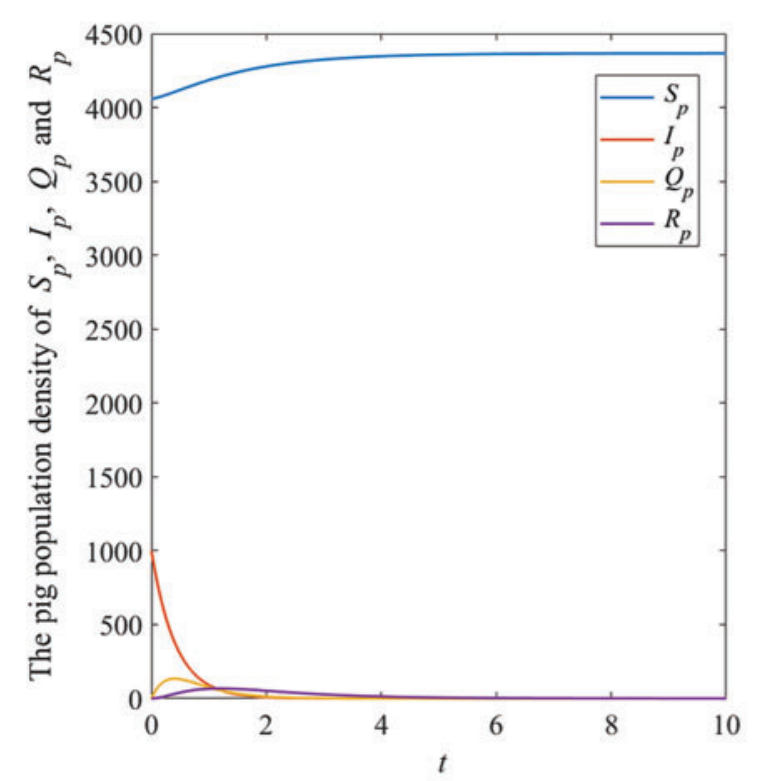

(a)

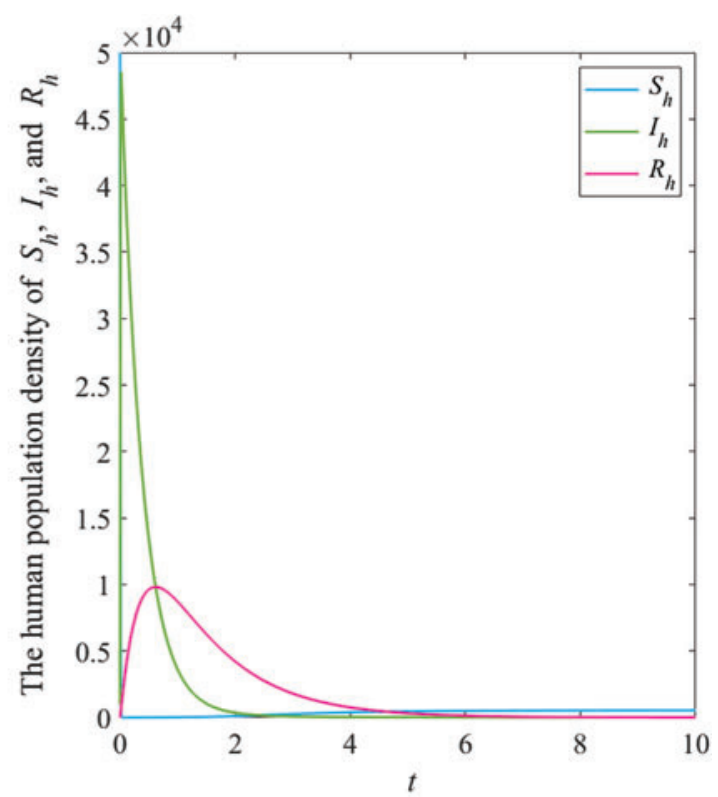

(b)

Figure 3: The simulation results of the system (1), (a) The plots of asymptotic population density of pig susceptible population $\left(S_{p}\right)$, pig infectious population $\left(I_{p}\right)$, pig isolated population $\left(Q_{p}\right)$, and pig recovery population $\left(R_{p}\right)$ with respect to time $t$ tends to the equilibrium $\left(E_{1}\right)$, and (b) The plots of asymptotic population density of human susceptible population $\left(S_{h}\right)$, human infectious population $\left(I_{h}\right)$, and human recovery population $\left(R_{h}\right)$ with respect to time $t$ tends to the equilibrium $\left(E_{1}\right)$

The results showed that the number of pig infectious cases dramatically decreased in the first quarter. After that, the decreasing of pig infectious cases slowly decreased. Then, it tended to the equilibrium value. Fig. 3a, the number of pig susceptible population increased after infectious cases were recovered. In addition, the isolated population increased when the number of infectious cases increased to control the spread of the disease. Then, it decreased after the number of infectious cases decreased. In human case (Fig. 3b), the number of infectious populations increased in the first quarter, then the number increased and approached to the equilibrium number. Therefore, the parameters of this case provided that the disease died out.

The solution trajectories tend to the disease-free equilibrium $\left(E_{2}\right)$ which satisfy Theorem 2 with the remaining parameter values $\mu=0.6, \alpha=0.5, \gamma=0.4, M=0.3, a=0.7, \beta_{2}=0.1$, and $\delta=0.8$ as shown in Fig. 4. The calculated reproduction numbers of this case are $R_{01}=2.5833>1$ and $R_{02}=$ $0.2125<1$.

The results showed that the number of pig infectious cases dramatically decreased in the first quarter. After that, the decreasing of pig infectious cases slowly decreased. Then, it tended to the equilibrium value. The number of pig susceptible population increased after infectious cases decreased. In human case (Fig. 4b), the number of infectious populations dramatically increased in the first quarter, then it decreased and approached to the equilibrium number. Therefore, the result of this case indicated that the disease in pig died out while it still appeared in human. 


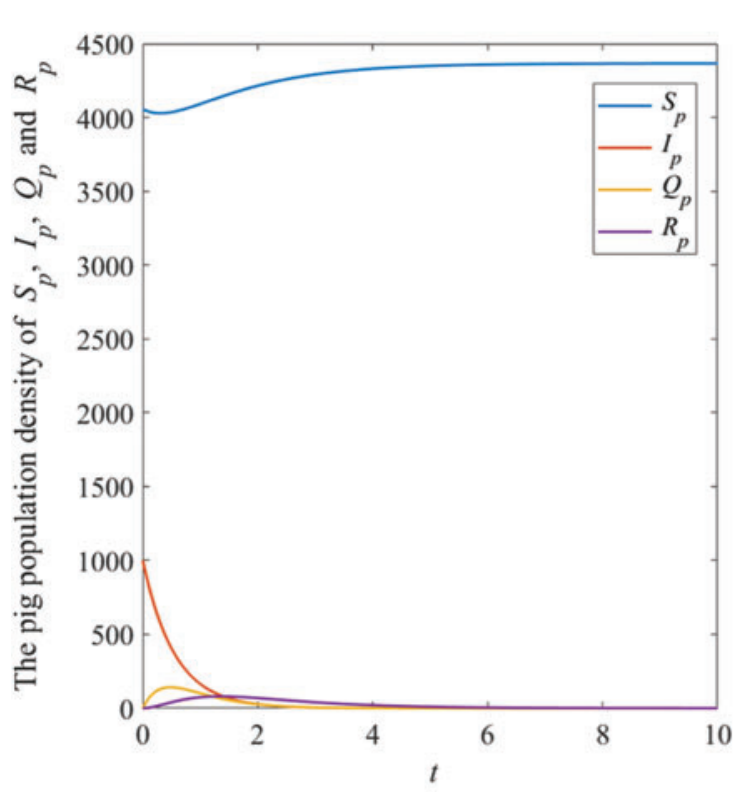

(a)

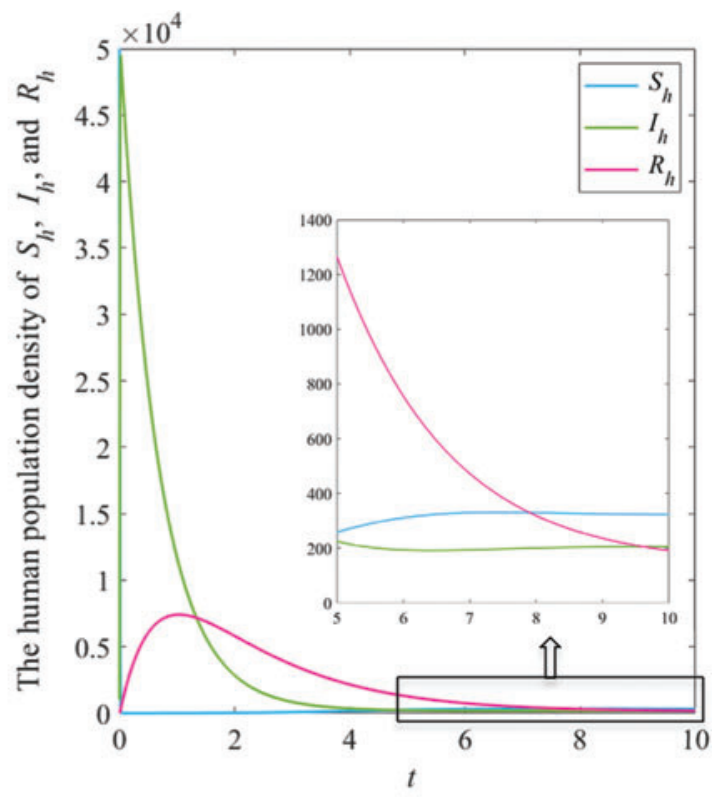

(b)

Figure 4: The simulation results of the system (1), (a) The plots of asymptotic population density of pig susceptible population $\left(S_{p}\right)$, pig infectious population $\left(I_{p}\right)$, pig isolated population $\left(Q_{p}\right)$, and pig recovery population $\left(R_{p}\right)$ with respect to time $t$ tends to the equilibrium $\left(E_{2}\right)$, and (b) The plots of asymptotic population density of human susceptible population $\left(S_{h}\right)$, human infectious population $\left(I_{h}\right)$, and human recovery population $\left(R_{h}\right)$ with respect to time $t$ tends to the equilibrium $\left(E_{2}\right)$

The numerical results of the system (1) with the remaining parameter values $\mu=0.5, \alpha=0.6, \gamma=$ $0.1, M=0.9, a=0.3, \beta_{2}=0.01$, and $\delta=0.1$ with $R_{01}=0.9568<1$ and $R_{02}=1.2473>1$. The solution trajectories tend to the endemic equilibrium $\left(E^{*}\right)$ which satisfy Theorem 3 as shown in Fig. 5. The number of pig susceptible population decreased in the first five quarters then it increased and approached to the equilibrium. The number of pig infectious population increase in the first quarter then it tends to equilibrium after five quarters. In human case, the number of human infectious population increased sharply in the first two quarters. After that, it decreased and tend to equilibrium value. The number of recovery population increased in the first quarter then it decreased and approached to equilibrium value. The result of this case showed that the disease still appeared in both human and pigs.

To consider the effect of moisture, the numerical results of the system (1) with the remaining parameter values $\mu=0.5, \alpha=0.6, \gamma=0.1, a=0.01, \beta_{2}=0.01$, and $\delta=0.01$. The solutions trajectories are computed with various values of relative humidity $M$ as $0.1,0.3,0.5,0.7$, and 0.9 is shown in Fig. 6.

In Fig. 6, the result indicates that when the relative humidity $(M)$ increases the pig infectious population density also increases. Particularly, the trajectories of pig infectious population decrease and approach to constant values when $M=0.1,0.3$, and 0.5 . On the other hand, the number of pig infectious population increase and go to equilibrium value when $M=0.7$ and 0.9. Moreover, when $M=0.1$ and 0.3 the trajectories approach zero. This means that the disease will die out when there is 
less moisture in the air. Therefore, the result confirms that there is an effect of the moisture in the air to the infection of Streptococcus suis as mentioned in the literature.

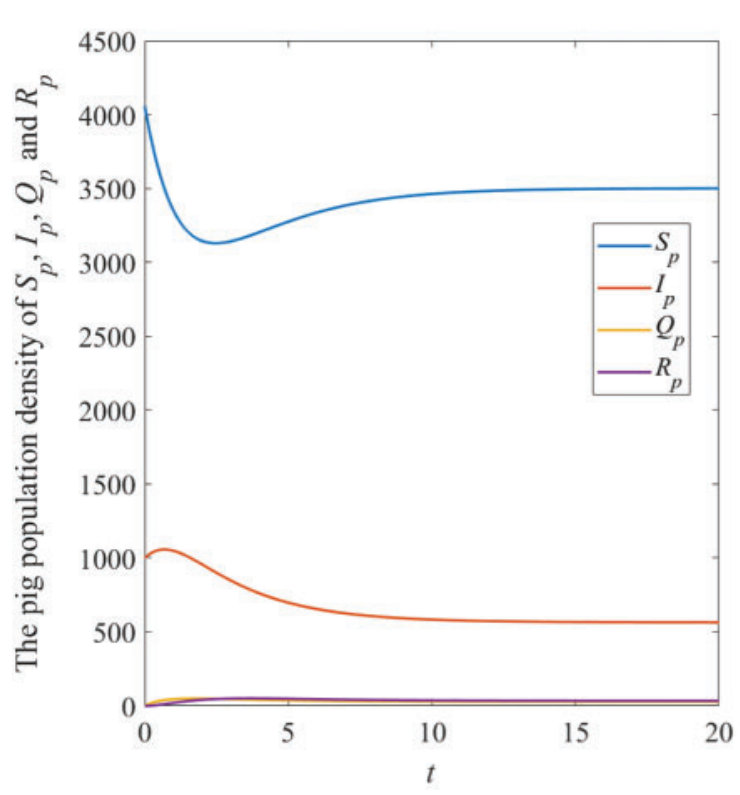

(a)

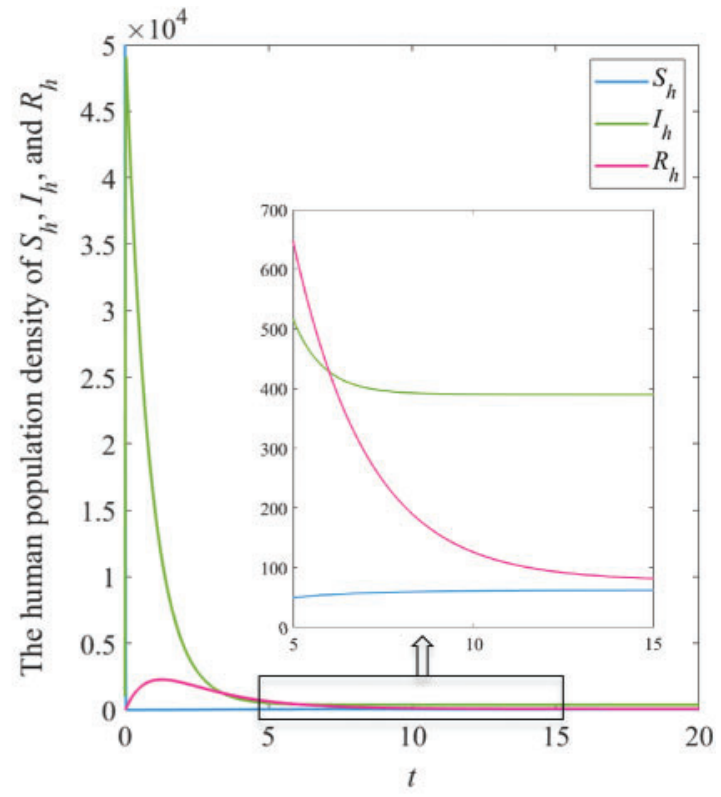

(b)

Figure 5: The simulation results of the system (1), (a) The plots of asymptotic population density of pig susceptible population $\left(S_{p}\right)$, pig infectious population $\left(I_{p}\right)$, pig isolated population $\left(Q_{p}\right)$, and pig recovery population $\left(R_{p}\right)$ with respect to time $t$ tends to the equilibrium $\left(E^{*}\right)$, and (b) The plots of asymptotic population density of human susceptible population $\left(S_{h}\right)$, human infectious population $\left(I_{h}\right)$, and human recovery population $\left(R_{h}\right)$ with respect to time $t$ tends to the equilibrium $\left(E^{*}\right)$

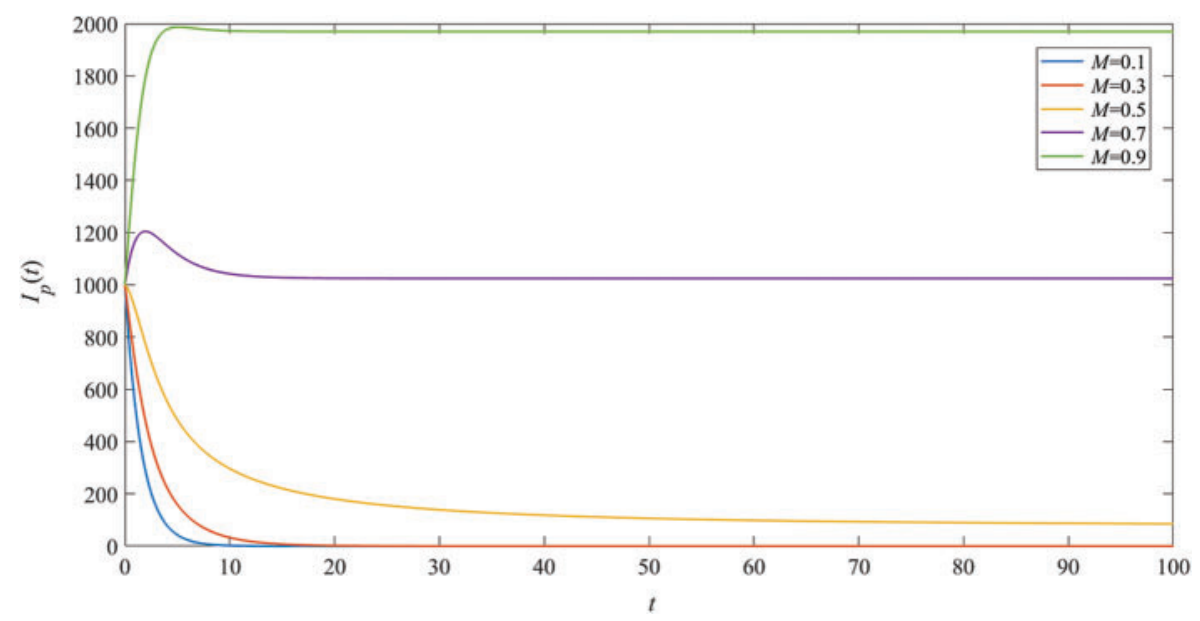

Figure 6: The simulation results of the pig infectious population $\left(I_{p}\right)$ for various values of relative humidity $M$ with respect to time $t$ 


\section{Conclusions}

We have proposed the mathematical model for predicting the disease transmission of Streptococcus suis between pigs and humans, which is developed from the SIQR model that considered only in the pig population. The pigs-human epidemiology model of Streptococcus suis transmission which is SIQR-SIR model is investigated. The model combined with susceptible $\left(S_{p}\right)$, infection $\left(I_{p}\right)$, isolation $\left(Q_{p}\right)$, and recovery $\left(R_{p}\right)$ for pig population and susceptible $\left(S_{h}\right)$, infectious $\left(I_{h}\right)$, and recovery $\left(R_{h}\right)$ for human population. Moreover, we studied the effect of moisture in the air on disease transmission. The equilibria of the model were analyzed. Then, the next-generation matrix was used to find the basic reproduction numbers $R_{01}$, and $R_{02}$. We obtained the conditions of the equilibrium points $E_{1}, E_{2}$, and $E^{*}$ which are locally asymptotically stable in Theorem 1 , Theorem 2 , and Theorem 3 , respectively. The results show that if $R_{01}<1$ and $R_{02}<1$, the equilibrium points $E_{1}$ is locally asymptotically stable while $E_{2}$ is locally asymptotically stable if $R_{01}>1$ and $R_{02}<1$. Finally, the endemic equilibrium $\left(E^{*}\right)$ is locally asymptotically stable if $R_{01}<1$ and $R_{02}>1$. The bifurcation diagram is also presented. The numerical examples confirmed the results of Theorems. The results indicated that moisture affects disease transmission. When the air has higher moisture the transmission of the pathogen is also high. Moreover, the model can be improved by considering infection of newborn pigs from infectious adult female pigs.

Acknowledgement: This research was financially supported by the young researcher development project of Khon Kaen University. Appreciation is extended to Faculty of Science, Khon Kaen University, Khon Kaen, Thailand.

Funding Statement: This research was financially supported by the young researcher development project of Khon Kaen University.

Conflicts of Interest: The authors declare that they have no conflicts of interest to report regarding the present study.

\section{References}

[1] K. E. Jones, N. G. Patel, M. A. Levy, A. Storeygard, D. Balk et al., "Global trends in emerging infectious diseases," Nature, vol. 451, pp. 990-993, 2008.

[2] F. Li and L. Du, "MERS coronavirus: An emerging zoonotic virus," Viruses, vol. 11, no. 7, pp. 663, 2019.

[3] J. S. Mackenzie and D. W. Smith, "COVID-19: A novel zoonotic disease caused by a coronavirus from China: What we know and what we don't," Microbiology Australia, vol. 41, no. 1, pp. 45-50, 2020.

[4] Z. R. Lun, Q. P. Wang, X. G. Chen, A. X. Li and X. Q. Zhu, "Streptococcus suis: An emerging zoonotic pathogen," The Lancet. Infectious Diseases, vol. 7, no. 3, pp. 201-209, 2011.

[5] H. J. Huh, K. J. Park, J. H. Jang, M. Lee, J. H. Lee et al., "Streptococcus suis meningitis with bilateral sensorineural hearing loss," The Korean Journal of Laboratory Medicine, vol. 31, no. 3, pp. 205-211, 2011.

[6] Y. Feng, H. Zhang, Z. Wu, S. Wang, M. Cao et al., "Streptococcus suis infection," Virulence, vol. 5, no. 4, pp. 477-497, 2014.

[7] M. Gajdács, A. Németh, M. Knausz, I. Barrak, A. Stájer et al., "Streptococcus suis: An underestimated emerging pathogen in Hungary?, " Microorganisms, vol. 8, no. 9, pp. 1292, 2020.

[8] N. Dekker, A. Bouma, I. Daemen, D. Klinkenberg, L. van Leengoed et al., "Effect of spatial separation of pigs on spread of streptococcus suis serotype 9, ” PLOS ONE, vol. 8, no. 4, pp. e61339, 2013.

[9] E. Giang, B. M. Hetman, J. M. Sargeant, Z. Poljak and A. L. Greer, "Examining the effect of host recruitment rates on the transmission of streptococcus suis in nursery swine populations," Pathogens, vol. 9, no. 3, pp. 174, 2020. 
[10] M. Okura, M. Osaki, R. Nomoto, S. Arai, R. Osawa et al., "Current taxonomical situation of streptococcus suis," Pathogens, vol. 5, no. 3, pp. 45, 2016.

[11] G. Goyette-Desjardins, J. Auger, J. Xu, M. Segura and M. Gottschalk, "Streptococcus suis, an important pig pathogen and emerging zoonotic agent - an update on the worldwide distribution based on serotyping and sequence typing," Emerging Microbes and Infections, vol. 3, no. 1, pp. 1-20, 2014.

[12] J. Dutkiewicz, J. Sroka, V. Zajac, B. Wasinski, E. Cisak et al., "Streptococcus suis: A re-emerging pathogen associated with occupational exposure to pigs or pork products. part I-Epidemiology, "Annals of Agricultural and Environmental Medicine, vol. 24, no. 4, pp. 683-695, 2017.

[13] M. Gottschalk, J. Xu, C. Calzas and M. Segura, "Streptococcus suis: A new emerging or an old neglected zoonotic pathogen?," Future Microbiol, vol. 5, no. 3, pp. 371-391, 2010.

[14] K. Oishi, A. Kerdsin, S. Dejsirilert, P. Puangpatra, S. Sripakdee, "Genotypic profile of streptococcus suis serotype 2 and clinical features of infection in humans, Thailand," Emerging Infectious Diseases, vol. 17, no. 5, pp. 835-842, 2011.

[15] A. Kerdsin, D. Takeuchi, A. Nuangmek, Y. Akeda, M. Gottschalk et al., "Genotypic comparison between streptococcus suis isolated from pigs and humans in Thailand," Pathogens, vol. 9, no. 1, pp. 50, 2020.

[16] A. Kerdsin, K. Oishi, S. Sripakdee, N. Boonkerd, P. Polwichai et al., "Clonal dissemination of streptococcus suis serotype 14 in Thailand," Journal of Medical Microbiology, vol. 58, no. 11, pp. 1508-1513, 2009.

[17] A. Kerdsin, R. Hatrongjit, M. Gottschalk, D. Takeuchi, S. Hamada et al., "Emergence of streptococcus suis serotype 9 infection in humans," Journal of Microbiology, Immunology and Infection, vol. 50, no. 4, pp. 545-546, 2017.

[18] R. Hatrongjit, A. Kerdsin, M. Gottschalk, D. Takeuchi, S. Hamada et al., "First human case report of sepsis due to infection with streptococcus suis serotype 31 in Thailand," BMC Infectious Diseases, vol. 15, pp. 392, 2015.

[19] J. Dutkiewicz, V. Zajac, J. Sroka, B. Wasinski, E. Cisak et al., "Streptococcus suis: A re-emerging pathogen associated with occupational exposure to pigs or pork products. part II-pathogenesis," Annals of Agricultural and Environmental Medicine, vol. 25, no. 1, pp. 186-203, 2018.

[20] J. F. Moxnes and K. Hausken, "Mathematical modelling of acute virus influenza a infections," Mathematical and Computer Modelling of Dynamical Systems, vol. 18, no. 5, pp. 521-538, 2012.

[21] U. Panbaba and S. Garba, "Stability analysis and optimal control for yellow fever model with vertical transmission," International Journal of Applied and Computational Mathematics, vol. 6, pp. 105, 2020.

[22] D. Prathumwan, K. Trachoo and I. Chaiya, "Mathematical modeling for prediction dynamics of the coronavirus disease 2019 (COVID-19) pandemic, quarantine control measures," Symmetry, vol. 12, no. 9, pp. 1404, 2020.

[23] K. Liang, "Mathematical model of infection kinetics and its analysis for COVID-19, SARS and MERS," Infection, Genetics and Evolution, vol. 82, pp. 104306, 2020.

[24] M. Sohaib and Attaullah, "Mathematical modeling and numerical simulation of HIV infection model," Results in Applied Mathematics, vol. 7, pp. 100118, 2020.

[25] M. U. Rahman, M. Arfan, Z. Shah, P. Kumam and M. Shutaywi, "Nonlinear fractional mathematical model of tuberculosis (TB) disease with incomplete treatment under atangana-baleanu derivative," Alexandria Engineering Journal, vol. 60, no. 3, pp. 2845-2856, 2021.

[26] M. Arfan, K. Shah, A. Ullah, M. Shutaywi, P. Kumam et al., "On fractional order model of tumor dynamics with drug interventions under nonlocal fractional derivative," Results in Physics, vol. 21, pp. 103783, 2021.

[27] K. Shah, R. U. Din, W. Deebani, P. Kumam and Z. Shah, "On nonlinear classical and fractional order dynamical system addressing COVID-19," Results in Physics, vol. 24, pp. 104069, 2021.

[28] M. A. Almuqrin, P. Goswami, S. Sharma, I. Khan, R. S. Dubey et al., "Fractional model of ebola virus in population of bats in frame of atangana-baleanu fractional derivative," Results in Physics, vol. 26, pp. 104295, 2021.

[29] H. M. Srivastava, R. S. Dubey and M. Jain, "A study of the fractional-order mathematical model of diabetes and its resulting complications," Mathematical Methods in the Applied Sciences, vol. 42, pp. 4570-4583, 2019. 
[30] S. S. Alzaid, B. S. T. Alkahtani, S. Sharma and R. S. Dubey, "Numerical solution of fractional model of hiv-1 infection in framework of different fractional derivatives," Journal of Function Spaces, vol. 2021, pp. 6642957, 2021.

[31] Z. Shah, R. Jan, P. Kumam, W. Deebani and M. Shutaywi, "Fractional dynamics of HIV with source term for the supply of new CD4+ T-cells depending on the viral load via caputo-Fabrizio derivative," Molecules, vol. 26, pp. 1806, 2021.

[32] P. Magal and G. Webb, "The parameter identification problem for SIR epidemic models: Identifying unreported cases," Journal of Mathematical Biology, vol. 77, pp. 1629-1648, 2018.

[33] M. De la Sen, A. Ibeas, S. Alonso-Quesada and R. Nistal, "On a SIR model in a patchy environment under constant and feedback decentralized controls with asymmetric parameterizations," Symmetry, vol. 11, no. 3, pp. 430, 2019.

[34] M. De la Sen, A. Ibeas and R. Agarwal, "On confinement and quarantine concerns on an SEIAR epidemic model with simulated parameterizations for the COVID-19 pandemic," Symmetry, vol. 12, no. 10, pp. 1646, 2020.

[35] C. Shen, M. Li, W. Zhang, Y. Yi, Y. Wang et al., "Modeling transmission dynamics of streptococcus suis with stage structure and sensitivity analysis," Discrete Dynamics in Nature and Society, vol. 2014, pp. 432602, 2014.

[36] W. Anna, A. Anna, B. Annette, S. Marie, G. Bengt et al., "Streptococcus suis in Swedish grower pigs: Occurrence, serotypes, and antimicrobial susceptibility," Acta Veterinaria Scandinavica, vol. 62, pp. 36, 2020.

[37] S. Dee, A. Carlson, N. Winkelman and M. Corey, "Effect of management practices on the streptococcus suis carrier rate in nursery swine," Journal of the American Veterinary Medical Association, vol. 203, no. 2, pp. 295-299, 1993.

[38] G. Zou, J. Zhou, R. Xiao, L. Zhang, Y. Cheng et al., "Effects of environmental and management-associated factors on prevalence and diversity of streptococcus suis in clinically healthy pig herds in China and the United Kingdom," Applied and Environmental Microbiology, vol. 84, no. 8, pp. e02590-17, 2018.

[39] T. M. Chen, J. Rui, Q. P. Wang, Z. Y. Zhao, J. A. Cui et al., "A mathematical model for simulating the phase-based transmissibility of a novel coronavirus," Infectious Diseases of Poverty, vol. 9, pp. 24, 2020.

[40] P. van den Driessche and J. Watmough, "Reproduction numbers and sub-threshold endemic equilibria for compartmental models of disease transmission," Mathematical Biosciences, vol. 180, no. 1-2, pp. 29-48, 2002.

[41] O. Diekmann, J. A. P. Heesterbeek and M. G. Roberts, "The construction of next-generation matrices for compartmental epidemic models," The Royal Society Interface, vol. 7, no. 47, pp. 873-885, 2009.

[42] Statista, Thailand: Birth rate from 2008 to 2018 (per 1,000 inhabitants). Statista, 2020. [Online]. Available: https://www.statista.com/statistics/977294/crude-birth-rate-inthailand/. 